Case Reports
in Dermatology

\title{
Sweet Syndrome and Secondary Syphilis in a Person with Acute Necrotizing Tonsillitis
}

\author{
Joseph Mishal ${ }^{a, b}$ Igor Viner ${ }^{b, c}$ Alexandro Livoff ${ }^{b, d}$ Shlomo Maayan ${ }^{\text {b, e }}$ \\ Eli Magen ${ }^{b}, f$
}

aMedicine B Department, Barzilai University Medical Center, Ashkelon, Israel; ${ }^{b}$ Ben-Gurion University of the Negev, Beersheba, Israel; 'Department of Emergency Medicine, Barzilai University Medical Center, Ashkelon, Israel; dPathology Department, Barzilai University Medical Center, Ashkelon, Israel; eDepartment of Infectious Diseases, Barzilai University Medical Center, Ashkelon, Israel; fMedicine C Department, Allergy and Clinical Immunology Division, Barzilai University Medical Center, Ashkelon, Israel

\section{Keywords}

Sweet syndrome $\cdot$ Secondary syphilis $\cdot$ Tonsillitis

\begin{abstract}
Syphilis has received its classical designation as one of "the great imitators," reflecting a wide variety of symptoms and presentations, which can cause difficulties in diagnosis. Here we report an unusual case of secondary syphilis in a person with acute necrotizing tonsillitis and Sweet syndrome. A 33-year-old female presented with fever, bilateral cervical lymphadenopathy, tonsillar enlargements with ulcerated pus-filled lesions on the right tonsil, and multiple pseudovesicular, mammillated, edematous plaques on her neck, face, and extremities. Syphilis serology was positive and a skin biopsy demonstrated a neutrophil-rich dermatitis characteristic of Sweet syndrome. The association of Treponema pallidum infection with Sweet syndrome may be a coincidence; nevertheless, our case serves as a reminder that secondary syphilis should remain in the differential diagnosis of the acute febrile neutrophilic dermatosis.
\end{abstract}




\section{Case Reports in Dermatology}

\section{Introduction}

Oropharyngeal/tonsillar chancres of primary syphilis have been previously reported in the literature, but these lesions are rare manifestations in later stages of syphilis. Secondary syphilis characteristically presents with cutaneous lesions affecting the skin and mucous membranes with or without genital involvement. Our report describes an unusual case of secondary syphilis in a patient who presented with tonsillitis and Sweet syndrome.

\section{Case Presentation}

A 33-year-old female presented to the emergency department after 2 weeks of painful sore throat, fever, chills, malaise, and a pruritic rash initially on her face and arms that spread to the neck and legs. She denied conjunctivitis, arthralgia, or other associated symptoms. She had no significant past medical history and was not taking any medication. The patient had been treated in the community with a 7-day course of amoxicillin for potential bacterial tonsillitis. As her sore throat did not improve, amoxicillin was changed to cefuroxime and roxithromycin for an additional 7 days of treatment without clinical improvement and the patient was referred to our hospital.

Physical examination findings were notable for a temperature of $37.9^{\circ} \mathrm{C}$, bilateral cervical lymphadenopathy, grade 3 tonsillar enlargements with ulcerated pus-filled lesions on the right tonsil (Fig. 1), and multiple pseudovesicular, mammillated, edematous plaques on her neck, face, and extremities (Fig. 2). A complete blood cell count revealed leukocytosis: white

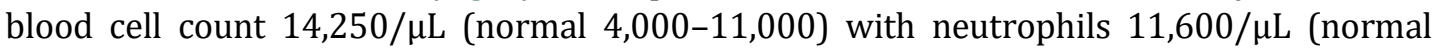
2,000-7,000). Throat, nasopharyngeal, blood, and urine cultures were negative (including Neisseria gonorrhoeae and Chlamydia trachomatis). Viral screening was negative for herpes simplex virus 1, herpes simplex virus 2, Epstein-Barr virus, cytomegalovirus, and H1N1 using PCR techniques. Throat cultures for detecting group A beta-hemolytic Streptococcus pyogenes and serum test for antistreptolysin- 0 antibodies were negative.

Syphilis serology demonstrated a Venereal Disease Research Laboratory test result of 1:32, a positive treponemal IgM/IgG antibody test, and a positive Treponema pallidum hemagglutination test. HIV p24 antibody and antigen tests were negative.

A skin biopsy specimen demonstrated a neutrophil-rich and acanthotic epidermis; a diffuse cellular infiltration of the upper dermis with a nodular tendency consisted of centrally located neutrophils, showing leukocytoclasia with admixed histiocytes and lymphocytes, plasma cells and eosinophils were absent; endothelial cell swelling and proliferation was noted but without extravasated red cells, fibrinoid necrosis of vessels, or microthrombi (Fig. 3). No microorganisms were visualized. The histological features were characteristic of Sweet syndrome, while according to the clinical and laboratory features a diagnosis of secondary syphilis was made. The patient was treated with weekly intramuscular injections of 2.4 million units of benzathine penicillin over a period of 4 weeks, followed by treatment with tapering doses of systemic prednisone $(1 \mathrm{mg} / \mathrm{kg}$ /day which were gradually tapered within 2 weeks). The tonsillar lesions and the rash resolved without scarring, but with little residual pigmentation.

\section{Karger'=}




\section{Case Reports in Dermatology}

\section{Discussion}

Syphilis, an infectious disease caused by the spirochete T. pallidum, has been a reemerging infectious disease in developed countries in the past decade [1]. Syphilis has received its classical designation as one of "the great imitators," reflecting a wide variety of symptoms and presentations, which can cause difficulties in diagnosis. To our knowledge, our case represents the first report of a coincidence of syphilitic tonsillitis and acute febrile neutrophilic dermatosis in secondary syphilis.

Syphilitic tonsillitis is a very rare manifestation in secondary syphilis [2-4]. In one recently published case report where the diagnosis was established based only on clinical manifestations and serological test results, the patient with syphilitic tonsillitis also had skin and perioral lesions [5]. In our case, syphilitic tonsillitis was also diagnosed on the basis of clinical manifestations and serological test results. Recently, Drago et al. [6] reported a similar case, though for the diagnosis of syphilitic tonsillitis they performed a tonsillar biopsy followed by a direct fluorescent antibody assay using rabbit polyclonal antiserum. Taking into consideration the invasive nature of tonsillar biopsy, we did not perform the procedure in our patient.

Acute febrile neutrophilic dermatosis was initially described in 1964 by Robert Douglas Sweet [7]. Although the pathogenesis of Sweet syndrome remains unknown, a hypersensitivity reaction to infectious or tumor antigens has been suggested [8]. The single previously reported case of secondary syphilis mimicking Sweet syndrome was described in 1986 by Jordaan and Cilliers [9]. Mucosal involvement of the mouth (buccal ulcerations, ulcerative stomatitis, recurrent aphthous ulcerations, and painful oral lesions) is uncommon in patients with classical Sweet syndrome [10]. Our patients did not have oral lesions but the clinical picture of acute necrotizing tonsillitis with cervical lymphadenopathy, which has not been reported in Sweet syndrome previously. Therefore, we suggest that the acute necrotizing tonsillitis was a manifestation of secondary syphilis rather than of Sweet syndrome.

The association of T. pallidum infection with Sweet syndrome may be a coincidence; nevertheless, our case serves as a reminder that secondary syphilis should remain in the differential diagnosis of the acute febrile neutrophilic dermatosis.

\section{Statement of Ethics}

The patient gave written informed consent for publication of her case (including publication of images).

\section{Conflict of Interest Statement}

The authors have no conflicts of interest to declare.

\section{Funding Sources}

There was no external funding.

\section{Karger'}




\section{Case Reports in Dermatology}

\section{Author Contributions}

All authors analyzed and interpreted the patient's clinical data, discussed the clinical case, and contributed to the final manuscript. All authors read and approved the final manuscript.

\section{References}

1 Patton ME, Su JR, Nelson R, Weinstock H; Centers for Disease Control and Prevention (CDC). Primary and secondary syphilis - United States, 2005-2013. MMWR Morb Mortal Wkly Rep. 2014 May;63(18):402-6.

2 Baarsma EA, Kazzaz B, Soei KI. Secondary syphilis of the tonsils. J Laryngol Otol. 1985 Jun;99(6):601-3.

3 Shimizu T, Shinogi J, Majima Y, Sakakura Y. Secondary syphilis of the tonsil. Arch Otorhinolaryngol. 1989; 246(2):117-20.

4 Hamlyn E, Marriott D, Gallagher RM. Secondary syphilis presenting as tonsillitis in three patients. J Laryngol Otol. 2006 Jul;120(7):602-4.

5 Gedela K, Boag F. Syphilitic tonsillitis in primary care: a case report. Br J Gen Pract. 2012 Apr;62 (597):21920.

6 Drago F, Ciccarese G, Cogorno L, Tomasini CF, Cozzani EC, Riva SF, et al. Primary syphilis of the oropharynx: an unusual location of a chancre. Int J STD AIDS. 2015 Aug;26(9):679-81.

7 Sweet RD. An acute febrile neutrophilic dermatosis. Br J Dermatol. 1964 Aug-Sep;76(8-9):349-56.

8 Cohen PR. Sweet's syndrome - a comprehensive review of an acute febrile neutrophilic dermatosis. Orphanet J Rare Dis. 2007 Jul;2(1):34

9 Jordaan HF, Cilliers J. Secondary syphilis mimicking Sweet's syndrome. Br J Dermatol. 1986 Oct;115(4):4956.

10 Notani K, Kobayashi S, Kondoh K, Shindoh M, Ferguson MM, Fukuda H. A case of Sweet's syndrome (acute febrile neutrophilic dermatosis) with palatal ulceration. Oral Surg Oral Med Oral Pathol Oral Radiol Endod. 2000 Apr;89(4):477-9. 


\section{Case Reports in Dermatology}

Case Rep Dermatol 2021;13:216-221

DOI: $10.1159 / 000509374$

(C) 2021 The Author(s). Published by S. Karger AG, Basel www.karger.com/cde

Mishal et al.: Sweet Syndrome and Secondary Syphilis in a Person with Acute Necrotizing Tonsillitis

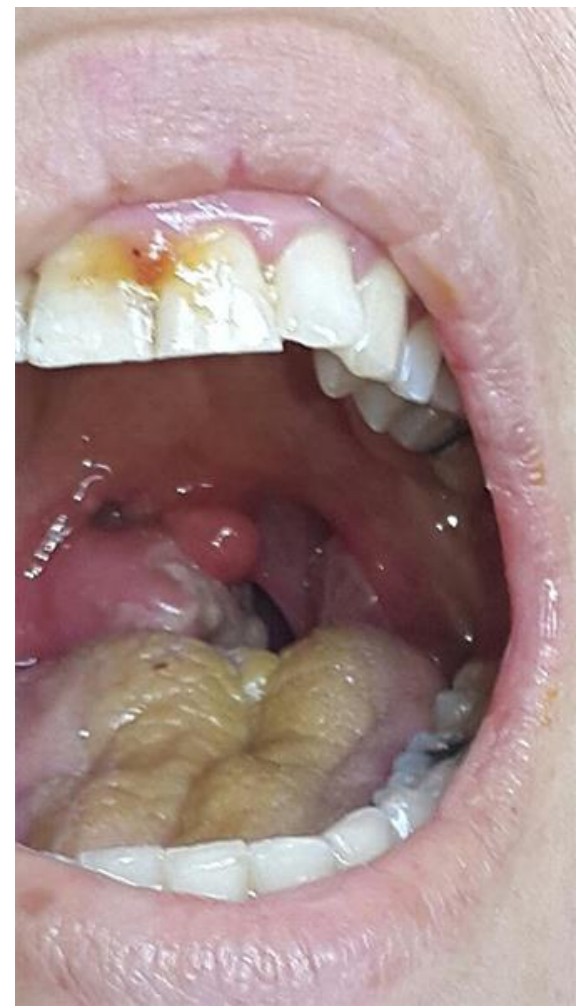

Fig. 1. Grade 3 tonsillar enlargements with ulcerated pus-filled lesions on both tonsils on hospital day 3 in a Treponema pallidum-infected woman with Sweet syndrome. 


\section{Case Reports in Dermatology}

Case Rep Dermatol 2021;13:216-221

DOI: $10.1159 / 000509374$

(c) 2021 The Author(s). Published by S. Karger AG, Basel www.karger.com/cde

Mishal et al.: Sweet Syndrome and Secondary Syphilis in a Person with Acute Necrotizing Tonsillitis

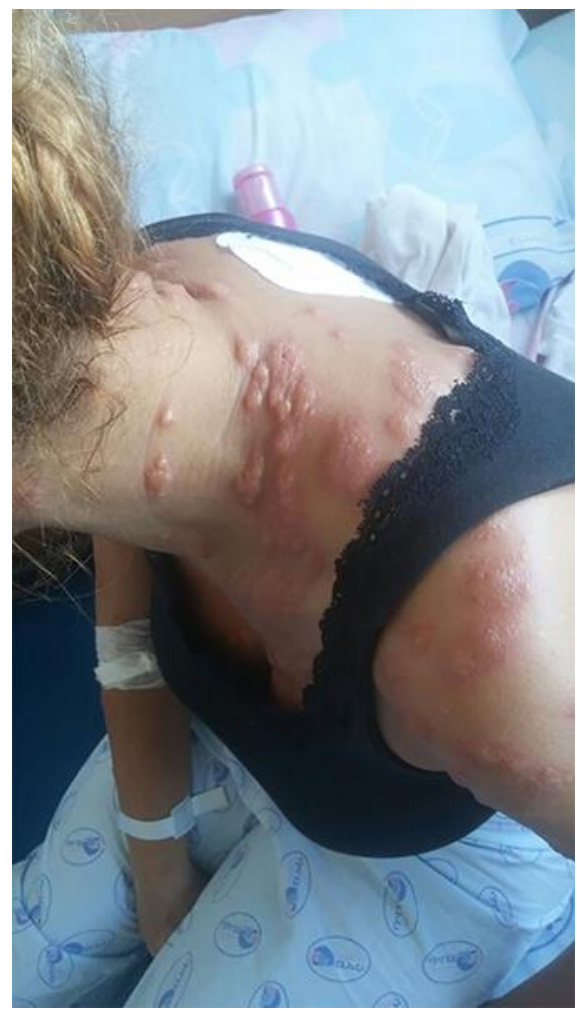

Fig. 2. Skin lesions on hospital day 3.
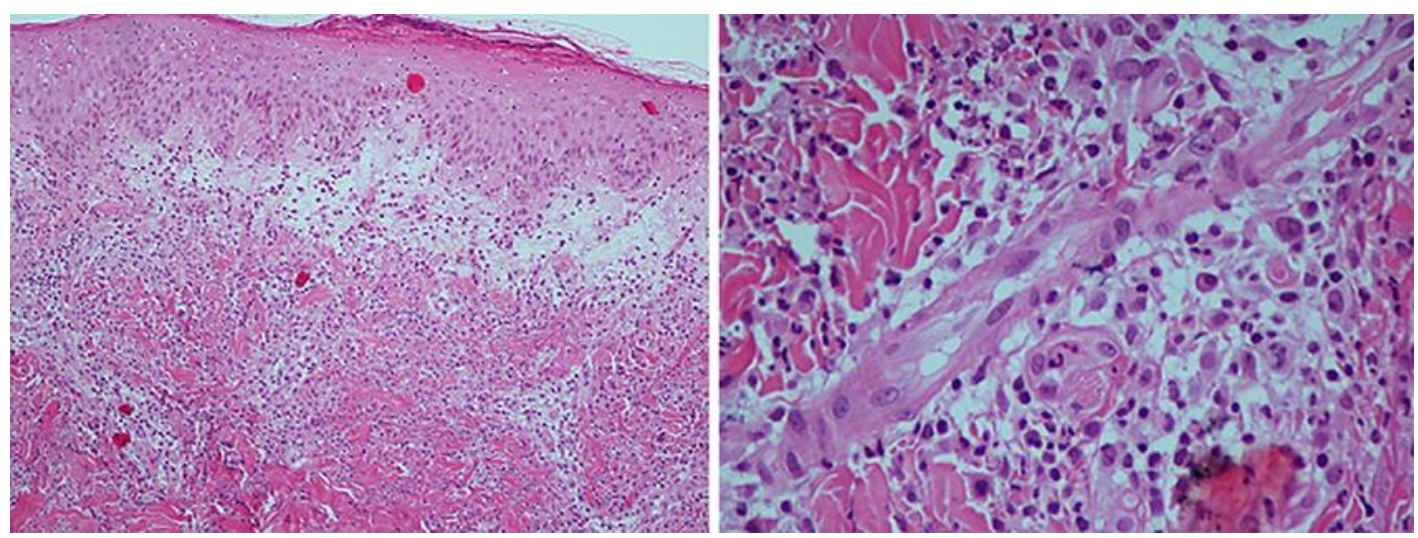

Fig. 3. Histopathology showing neutrophil-rich upper dermal infiltrate.

\section{Karger'}

